\title{
A rare etiology of persistent jaundice in type 1 autoimmune hepatitis
}

\author{
Budumuri Gautam V. Kumar, Purna Ch. Sethy, Rohit Gupta* \\ Department of Medical Gastroenterology, All India Institute of Medical Sciences, Rishikesh, India
}

\begin{abstract}
A 24-year-old male without previously known comorbidities presented with progressive jaundice and hepatomegaly. Autoimmune serology and liver biopsy revealed features of autoimmune hepatitis. A treatment by oral prednisolone and azathioprine was initiated, after which he achieved remission. However, hyperbilirubinemia persisted, with a predominantly unconjugated fraction. Hemolytic causes of unconjugated hyperbilirubinemia were ruled out, and the diagnosis of Gilbert syndrome was established.

Keywords: autoimmune hepatitis; hyperbilirubinemia; Gilbert syndrome
\end{abstract}

\section{Introduction}

Autoimmune hepatitis (AlH) is a chronic inflammatory liver disease, characterized by immune-mediated hepatocellular injury. This condition is seen across all age groups with a higher predilection for the female sex $[1,2]$. Although the clinical presentation is highly inconstant, AlH usually manifests as hepatocellular jaundice with elevated transaminase and constitutional symptoms. AlH can also present as overlap syndromes, associating characteristics of primary biliary cholangitis (PBC), primary sclerosing cholangitis (PSC), and cholestatic hepatitis. These overlap syndromes are suspected in patients having additional cholestatic symptoms, abnormal cholangiograms, or a positive antimitochondrial antibody (AMA) serology [3]. The presence of unconjugated hyperbilirubinemia in AlH patients suggests either simultaneously associated hemolysis or

Received: August 2021; Accepted after review: September 2021; Published: September 2021.

${ }^{*}$ Corresponding author: Rohit Gupta, Additional Professor and Head of Department of Medical Gastroenterology, All India Institute of Medical Sciences (AlIMS), Rishikesh, India- 249203.

Email: docgupta1976@gmail.com familial causes of hyperbilirubinemia. We present the case of a patient diagnosed with type $1 \mathrm{AlH}$, in whom remission of the disease was obtained with glucocorticoids, but with clinical persistence of jaundice. On thorough workup for the etiology, he was found to have Gilbert syndrome.

\section{Case report}

A 24-year-old male with no significant previous medical history presented with progressive hepatocellular jaundice and dull aching right upper quadrant pain for two months. He also had a history of anorexia and mild fatigability. However, he denied having any history of viral prodrome, alcohol abuse, recent travel, prior blood transfusions, recreational drugs, complementary medication use, or multiple sexual partners. Further, he denied any family history of liver disease. Except for icterus and hepatomegaly, other physical findings were insignificant. Laboratory findings were noteworthy for abnormal liver function tests in the form of raised AST (244 $\mathrm{U} / \mathrm{L}) / \mathrm{ALT}(311 \mathrm{U} / \mathrm{L})$ and total bilirubin of 28 $\mathrm{mg} / \mathrm{dl}$ with a predominant conjugated fraction $(18 \mathrm{mg} / \mathrm{dl})$, elevated alkaline phosphatase 
(245 U/L), hypergammaglobulinemia (4500 $\mathrm{mg} / \mathrm{dl}$ ) and coagulopathy (Table 1). Abdominal ultrasonography and magnetic resonance cholangiopancreatography were unremarkable. An extensive workup was done to find the etiology of his elevated liver enzymes. Serology for hepatotropic viruses (HBV, HCV, HEV, and HAV), Wilson disease, and hemochromatosis workup was negative. His serological markers for autoimmune hepatitis as antinuclear antibody (ANA) and smooth muscle antibody (SMA) were positive. However, additional markers as anti-liver kidney microsome (LKM) type 1, anti-liver cytosol antibody type 1 (anti-LC1) and antimitochondrial antibodies (AMA) were negative. Liver biopsy demonstrated lymphoplasmacytic infiltrate with marked interface activity and multiple areas of confluent necrosis consistent with type 1 autoimmune hepatitis (Figure 1). The patient received a combination of prednisone $(30 \mathrm{mg})$ and azathioprine $(50 \mathrm{mg})$. He improved over the next 3 to 4 weeks with normalization of transaminases and total gamma globulin levels. However, his total bilirubin was persistently elevated (total bilirubin $>5 \mathrm{mg} / \mathrm{dl}$ ), with a predominant unconjugated fraction. We further evaluated him for hemolytic causes of unconjugated hyperbilirubinemia, including hemoglobinopathies, which all turned out to be negative. Due to his young age, male sex and unconjugated hyperbilirubinemia, Gilbert syndrome was considered, and genotyping showed decreased enzymatic activity of UDP Glucuronosyl transferase Family 1 Member A Complex Locus (UGT1A) gene polymorphism.

Table 1. Biochemical parameters

\begin{tabular}{|c|c|c|c|c|c|c|c|}
\hline INVESTIGATION & Day 1 & Day 7 & Day 14 & Day 21 & Day 45 & Day 60 & Day 90 \\
\hline $\mathrm{Hb}(13-15 \mathrm{~g} / \mathrm{dl})$ & 12.5 & 13.5 & & & 14.2 & & 13.9 \\
\hline $\begin{array}{l}\text { TLC } \\
\left(4000-7000 / \mathrm{mm}^{3}\right)\end{array}$ & 11200 & 9400 & & & 7900 & & 8200 \\
\hline $\begin{array}{l}\text { Platelet count } \\
\left(150000-400000 / \mathrm{mm}^{3}\right)\end{array}$ & 349000 & 421000 & & & 389000 & & 375000 \\
\hline $\begin{array}{l}\text { Bilirubin }(\mathrm{T}) \\
(0.3-1.2 \mathrm{mg} / \mathrm{dl})\end{array}$ & 28 & 24 & 9.5 & 6.5 & 4.54 & 4.1 & 5.65 \\
\hline Bilirubin (D) & 18 & 12 & 4.3 & 0.89 & 0.33 & 0.48 & 0.45 \\
\hline $\begin{array}{l}\text { AST/ALT } \\
(0-40 \mathrm{U} / \mathrm{L})\end{array}$ & $244 / 311$ & $151 / 182$ & $114 / 160$ & $49 / 72$ & $38 / 42$ & $10 / 20$ & $11 / 20$ \\
\hline ALP $(30-240 \mathrm{U} / \mathrm{L})$ & 245 & 468 & 187 & 165 & & 141 & 156 \\
\hline GGT (0 - 55 U/L) & 38 & 24 & & & & 23 & 22 \\
\hline Protein $(6.4-8.3 \mathrm{mg} / \mathrm{dl})$ & 9.9 & 7.8 & 6.9 & & & 8.00 & 7.6 \\
\hline Albumin (3.5-5 mg/dl) & 4.2 & 4.4 & 3.8 & & & 4.6 & 5.0 \\
\hline $\begin{array}{l}\text { Total IgG levels } \\
\text { (<1600 mg/dl) }\end{array}$ & 4500 & & & & & & 899 \\
\hline
\end{tabular}

*Hb: Hemoglobin, TLC: Total Leucocyte Count, AST: aspartate transaminase, ALT: alanine transaminase, ALP: Alkaline Phosphatase, GGT: Gamma-Glutamyl Transferase 

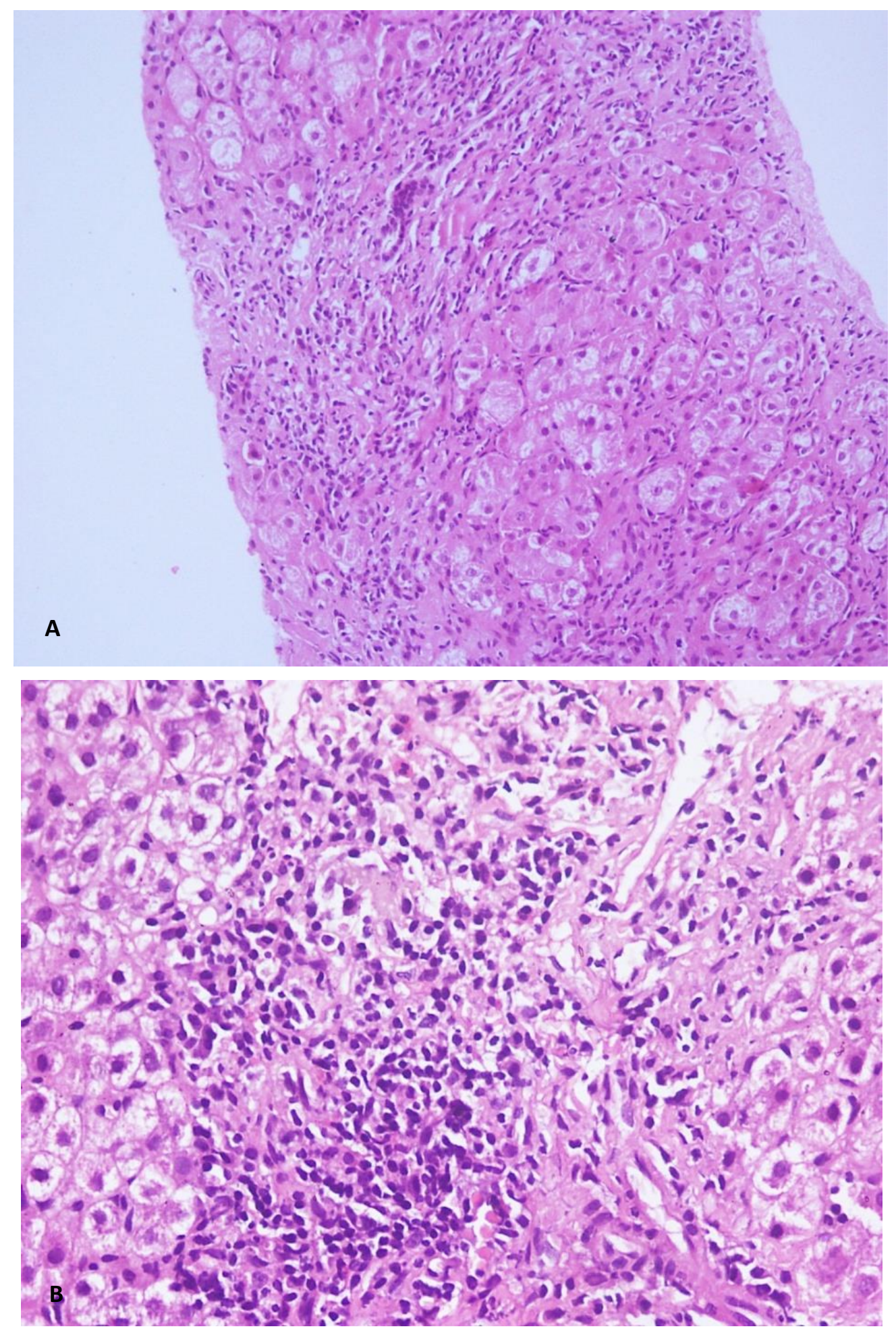

Fig. 1: Microscopical aspect of liver biopsy: hydropic change of hepatocytes, enlarged portal spaces with interface hepatitis (abundant lymphoplasmacytic inflammatory infiltrate) (HE, x200 - A; x400 - B)

\section{Discussion}

Autoimmune Hepatitis (AlH) was first described in 1951 [4] as a chronic progressive inflammatory liver disease of unknown etiology, causing immune-mediated hepatocyte injury [5]. It is characterized by the presence of interface hepatitis with lymphoplasmacytic infiltration on histology, hypergammaglobulinemia, and seropositive autoantibodies [6]. AlH is classified into three subtypes (type I, II, and III) based on 
distinctive serologic profiles [7]. Of these, type $\mathrm{I} \mathrm{AlH}$ is the most common subtype [8]. Antibodies to ANA, SMA, LKM type 1, and anti-SLA are diagnostic serologic markers with low sensitivity but high specificity. Other autoantibodies, including Anti-F-actin b, AntiLKM 3, Anti-Asialoglycoprotein receptor (ASGPR), and anti-LC1, are also present in some patients, but they are tested less frequently.

Clinical features are variable and can also reflect the activity of liver disease. Patients with mild fatigability, hepatocellular jaundice, and hepatomegaly usually have active disease flare. The presence of ascites and encephalopathy suggests underlying cirrhosis. Diagnosis of $\mathrm{AlH}$ is made by Simplified Diagnostic Criteria (SDC) validated by the International Autoimmune Hepatitis Group (IAIHG) in 2008 [9]. With a SDC score of $6 \mathrm{AlH}$ is considered probable, while a score of 7 is considered diagnostic for $\mathrm{AlH}$. In our index case, this score was 7 .

Therapy is indicated to all AlH patients. The primary goal of treatment is to achieve remission. Glucocorticoids with or without azathioprine remain the mainstay of treatment in the setting of disease flares [5]. Both prednisolone monotherapy and the combination of prednisolone with azathioprine are equivalent in efficacy for induction treatment. Azathioprene in combination with low dose prednisone (5-10 $\mathrm{mg} /$ day) is used as maintenance therapy [10].

Prognosis is determined by early diagnosis and therapy. Approximately $40 \%$ of severe untreated patients die within six months, while $50-90 \%$ relapse within a year [11]. The levels of AST, ALT and gamma globulin in the blood reflect the severity of the disease and correlates with the prognosis.

Coexistence of jaundice with predominantly unconjugated hyperbilirubinemia includes several pathophysiological mechanisms It can be due to increased bilirubin biosynthesis, aberrant bilirubin metabolism (uptake and conjugation), or both. Hemolysis is also frequent in AlH. Both Coomb's positive and Coomb's negative autoimmune hemolytic anemia are conceivable due to polyautoimmunity and splenomegaly. When overt hemolytic causes are ruled out, familial unconjugated hyperbilirubinemias such as Gilbert and Crigler-Najjar syndromes should be considered.

Gilbert syndrome (GS) is a genetically acquired autosomal recessive disorder that can trigger indirect hyperbilirubinemia episodes. GS is characterized by reduced UGT1A1 enzyme activity due to additional thymine-adenine (TA) repeats in the TATAA element in the promoter region of the UGT1A1 gene [12]. It is present in up to $10 \%$ of the general population and does not require therapy [12, 13]. So, in any young patient presenting with asymptomatic unconjugated hyperbilirubinemia, GS can be diagnosed by measuring UGT1A1 polymorphism. As far as we know, a link between GS and autoimmune hepatitis or other autoimmune diseases has not been reported in the literature. GS remains undetected throughout life until it is triggered by sepsis, medications, or stress [12, 14]. In our case, GS may have been triggered by the underlying autoimmune hepatitis flare or vice versa. More studies are needed to establish the exact relationship.

This patient is currently doing well and has achieved clinical remission; he received the recommendation to do a complete blood count, liver function tests and measure gamma globulin level every three months. Abdominal ultrasonography was indicated for cirrhosis screening. Our further plan is to taper the steroids to low doses and continue azathioprine as maintenance therapy. Prognosis in our patient mainly depends on the duration of disease remission and compliance to drugs. As relapse is universal, careful monitoring of the above mentioned clinical and biochemical parameters will lead to the success of therapy.

\section{Conclusion}

Autoimmune hepatitis is characterized by a chronic immune-mediated liver injury, which is often complicated and needs the evaluation of several parameters.

Jaundice in $\mathrm{AlH}$ is predominantly hepatocellular, and any persistent cholestatictype liver injury predicts the coexistence of 
either Primary Biliary Cholangitis or Primary Sclerosing Cholangitis.

The predominance of unconjugated hyperbilirubinemia is uncommon, but when present, one must think of additional hemolytic causes or inherited syndromes such as Gilbert syndrome.

Gilbert syndrome is a frequent benign condition that causes indirect hyperbilirubinemia, which is of a cosmetic concern. Its coexistence with $\mathrm{AlH}$ is less reported.

\section{References}

1. Manns MP, Czaja AJ, Gorham JD, et al. Diagnosis and management of autoimmune hepatitis. Hepatology 2010; 51(6):2193-2213.

2. Czaja AJ, dos Santos RM, Porto $A$, Santrach PJ, Moore SB. Immune phenotype of chronic liver disease. Dig Dis Sci 1998; 43(9):21492155.

3. Bunchorntavakul C, Reddy KR. Diagnosis and management of overlap syndromes. Clin Liver Dis 2015; 19(1):81-97.

4. Waldenstrom J. Liver, blood proteins and nutritive protein. Dtsch Z Verdau Stoffwechselkr 1953; 9:113-119.

5. Manns MP, Lohse AW, Vergani D. Autoimmune hepatitis--Update 2015. J Hepatol 2015; 62(1):S100-S11.

6. Doumtsis P, Oikonomou T, Goulis I, Zachou K, Dalekos G, Cholongitas E. Type 1 autoimmune hepatitis presenting with severe autoimmune neutropenia. Ann Gastroenterol 2018; 31(1):123-126.

7. Czaja AJ. Diagnosis and Management of Autoimmune Hepatitis: Current Status and Future Directions. Gut Liver 2016; 10(2):177203.

8. Vierling JM. Autoimmune Hepatitis and Overlap Syndromes: Diagnosis and Management. Clin Gastroenterol Hepatol 2015; 13(12):2088-2108.

\section{Author contribution}

Conceptualization and initial draft preparation: Budumuri Gautam V Kumar. Manuscript editing: Purna Ch. Sethy and Rohit Gupta. The published version of the work has been reviewed and approved by all authors.

\section{Conflicts of interest}

There are no personal, financial, or other conflicts of interest to disclose.

\section{Consent for publication}

Not applicable for this article.

9. Hennes EM, Zeniya M, Czaja AJ, et al. Simplified criteria for the diagnosis of autoimmune hepatitis. Hepatology 2008; 48(1):169-176.

10. Lamers $M M H$, van Oijen $M G H$, Pronk $M$, Drenth JPH. Treatment options for autoimmune hepatitis: a systematic review of randomized controlled trials. J Hepatol 2010; 53(1):191198.

11. Mack CL, Adams D, Assis DN, et al. Diagnosis and management of autoimmune hepatitis in adults and children: 2019 practice guidance and guidelines from the American Association for the Study of Liver Diseases. Hepatology 2020; 72(2):671-722.

12. Wagner $K-H$, Shiels $R G$, Lang $C A$, Seyed Khoei N, Bulmer AC. Diagnostic criteria and contributors to Gilbert's syndrome. Crit Rev Clin Lab Sci 2018; 55(2):129-139.

13. Strassburg CP. Hyperbilirubinemia syndromes (Gilbert-Meulengracht, Crigler-Najjar, DubinJohnson, and Rotor syndrome). Best Pract Res Clin Gastroenterol 2010; 24(5):555-571.

14. Kamal S, Abdelhakam S, Ghoraba D, et al. The frequency, clinical course, and health related quality of life in adults with Gilbert's syndrome: a longitudinal study. BMC Gastroenterol 2019; 19(1):22. 